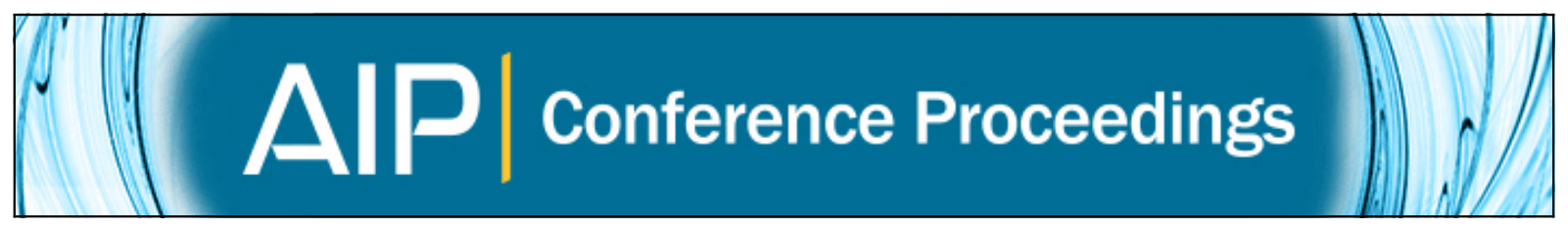

Cosmic ray experiments and the implications for indirect detection of dark matter

John W. Mitchell, Jonathan F. Ormes, and Robert E. Streitmatter

Citation: AIP Conference Proceedings 1516, 167 (2013); doi: 10.1063/1.4792562

View online: http://dx.doi.org/10.1063/1.4792562

View Table of Contents: http://scitation.aip.org/content/aip/proceeding/aipcp/1516?ver=pdfcov

Published by the AIP Publishing

Articles you may be interested in

Hoping to indirectly detect Dark Matter with cosmic rays

AIP Conf. Proc. 1304, 152 (2010); 10.1063/1.3527196

Dark Matter Solutions to the Cosmic Ray Lepton Puzzle

AIP Conf. Proc. 1241, 343 (2010); 10.1063/1.3462654

Cosmic-Ray Signatures of Dark Matter Decay

AIP Conf. Proc. 1200, 1055 (2010); 10.1063/1.3327537

Implications of 2d FGRS Results on Cosmic Structure

AIP Conf. Proc. 666, 275 (2003); 10.1063/1.1581805

Probing the cosmic dark age in X-rays

AIP Conf. Proc. 599, 140 (2001); 10.1063/1.1434627 


\title{
Cosmic Ray Experiments and the Implications for Indirect Detection of Dark Matter
}

\author{
John W. Mitchell ${ }^{\mathrm{a}}$, Jonathan F. Ormes ${ }^{\mathrm{b}}$ and Robert E. Streitmatter ${ }^{\mathrm{c}}$ \\ ${ }^{a}$ NASA Goddard Space Flight Center, Code 661, Greenbelt, MD 20771 \\ ${ }^{b}$ University of Denver, 2112 E. Wesley Ave., Denver, CO 80208 \\ ${ }^{c}$ NASA retired, 6505 Queens Chapel Road, University Park, MD 20782
}

\begin{abstract}
Detection of cosmic-ray antiprotons was first reported by Golden et al. in 1979 and their existence was firmly established by the BESS and IMAX collaborations in the early 1990s. Increasingly precise measurements of the antiproton spectrum, most recently from BESS-Polar and PAMELA, have made it an important tool for investigating cosmic-ray transport in the galaxy and heliosphere and for constraining dark-matter models. The history of antiproton measurements will be briefly reviewed. The current status will be discussed, focusing on the results of BESS-Polar II and their implications for the possibility of antiprotons from primordial black hole evaporation. The current results of the BESS-Polar II antihelium search are also presented.
\end{abstract}

Keywords: cosmic rays, antiprotons, antimatter, dark matter

PACS: $96.50 . \mathrm{S}-$, 96.50.Vg, 01.65.+g, 95.35.+d

\section{INTRODUCTION}

The cosmic radiation is dominated by matter but contains a small fraction of antiparticles that allow us to address important questions regarding the early history of the Universe and elementary particles. These include matter/antimatter asymmetry of the Universe and potential primary sources of cosmic rays from dark matter annihilation and primordial black holes (PBH). In addition, the antiparticles enable studies of fundamental cosmic ray phenomena such as their production and propagation in the interstellar medium, their transport into the solar system, modulation by entrained magnetic fields in the solar wind, and production in the atmosphere, all of which must be understood in order to know their sources.

Most cosmic ray antiprotons are produced by high-energy cosmic rays in p-p collisions in the interstellar medium where the some of the energy goes into the production of a proton-antiproton pair $(p+p->p+a n t i p r o t o n+p+p)$. The kinematics of this process imply a secondary spectrum that peaks at $\sim 2 \mathrm{GeV}$ and falls off steeply towards higher and lower energies. The antiproton/proton ratio is of order $10^{-5}$ at 1 $\mathrm{GeV}$. On top of this secondary spectrum a primary component might be observable as excess flux above or below the peak energy.

The observed dominance of matter likely results from symmetry breaking in the early universe. If this was local, small primordial antimatter domains could remain. The negligible probability of antinuclei with $|Z| \geq 2$ from cosmic ray interactions means that detecting even one such antinucleus would be a clear indication of such domains. 


\section{COSMIC-RAY ANTIPROTON CHRONOLOGY}

As noted in [1] "By 1963, practical magnetic spectrometers could be made with superconducting magnets. At this time Luis Alvarez suggested that [2], since lightweight magnetic spectrometers were feasible, they could be applied to high-energy cosmic ray experiments." His student Robert Golden reported [3] the first cosmic ray antiproton measurements. While this is usually credited as the discovery paper, that same year Bogomolov et al. [4] also reported identifying a few antiprotons using a small permanent magnet spectrometer. In 1981 Buffington reported an unexpectedly large flux of a few hundred antiprotons identified by their annihilation energy [5].

These scientific developments and the simultaneous planning for a large Space Station known as "Freedom" stimulated thinking in the US, Italy and Japan about a superconducting magnet spectrometer facility for the study of cosmic rays $[6,7,8,9]$ but this project was dropped when NASA scaled back plans for this facility.

Two subsequent experiments, PBAR and LEAP, cast large doubt on the measured flux of low energy antiprotons as being due to sources other than $p-p$ collisions $[10$, 11]. However, antiprotons had not yet been identified by mass found by simultaneously measuring charge, curvature in a magnetic field, and velocity. The first experiments to do so were IMAX [12] and the first flight of BESS [13]. Measurements by BESS, HEAT-pbar, CAPRICE, and AMS-01 (references in [14]) soon established that the bulk of the cosmic ray antiprotons were secondary in origin. The antiproton spectrum has now been precisely measured by BESS-Polar $[15,16]$ and PAMELA [17]. Additional improvements in statistics and upper energy range are expected from AMS-02.

\section{BESS RESULTS}

Successively improved versions of the BESS spectrometer were flown eleven times between 1993 and 2007 [18], including two long-duration Antarctic flights, obtaining ever more statistics on antiprotons. BESS measurements at solar minimum in 1995 and 1997 suggested the possibility of primary antiprotons from a source such as the evaporation of PBH [19]. The most recent result is given in Figure 1 showing 7886 antiprotons recorded by BESS-Polar II at solar minimum [16]. Careful comparison of
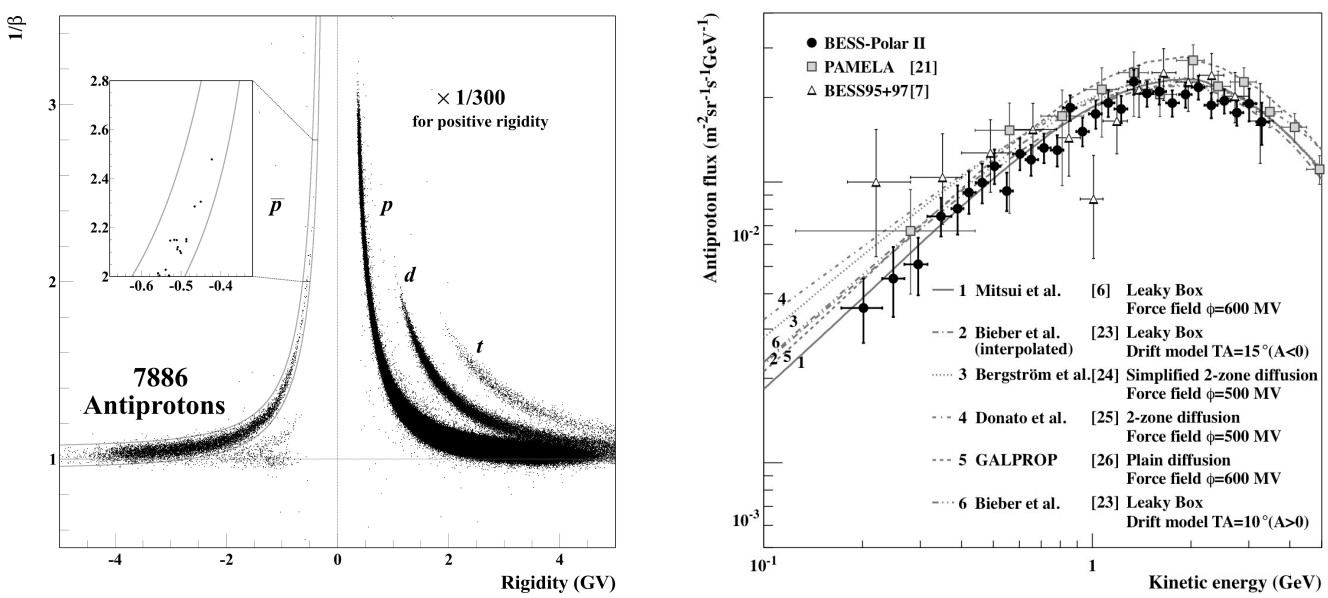

Figure 1: (a) left: A particle identification plot showing the clear separation of negative and positively charged low rigidity particles. (b) Right: Data from BESS and PAMELA compared to model spectra. 
the spectrum measured by BESS-Polar II to secondary models shows that within statistics there is no evidence of low-energy primary antiprotons.

BESS has also set new upper limits on antihelium in the cosmic rays [20]. If antihelium is assumed to have the same spectrum as helium, combining all BESS data gives a 95\% confidence upper limit of $6.9 \times 10^{-8}$ to the possible ratio of antihelium/helium from 1.0 to $14 \mathrm{GV}$. With no spectral assumption, the upper limit to this ratio is $1.0 \times 10^{-7}$ from 1.6 to $14 \mathrm{GV}$. Upper limits on heavier antinuclei were set by the C2 experiment on the HEAO-3 satellite [21]. BESS has also searched for antideuterium [22] and investigated solar modulation [23, 15].

\section{ACKNOWLEDGMENTS}

The authors gratefully acknowledge the contribution of all the members of the BESS collaboration who have contributed to these results. We are also grateful for the support of this program for many years by NASA through numerous grants.

\section{REFERENCES}

1. L. Smith, A. Buffington, M. Wahlig and P. Dauber, "A Superconducting Magnetic Spectrometer for Cosmic ray Nuclei”, Space Sciences Laboratory Series 12, Issue 47, Univ. of California, Berkeley, 1971.

2. L.W. Alvarez, "A Study of High Energy Interactions using a "Beam" of Primary Cosmic Ray Protons". Alvarez Physics Memo (503) 1964.

3. R.L. Golden et al., Phys. Rev. Lett. 43, 1196 (1979).

4. E.A. Bogomolov et al., 16th Int. Cosmic Ray Conf. (Kyoto) 1, 330 (1979).

5. A. Buffington, S.M. Schindler and C.R. Pennypacker, Astrophys. J. 248, 1179 (1981).

6. L.M. Barbier, E.R. Christian, J.F. Ormes, and G.F. Smoot, Nucl. Phys. B, Proc. Suppl. 14B, 3 (1990).

7. M.A. Green et al., "Astromag: A Superconducting Particle Astrophysics Magnet Facility for the Space Station", Proc. of IEEE Conf. on Applied Superconductivity, Baltimore, 1986.

8. J.F. Ormes, "Particle Astrophysics Magnet Facility for Space Station," Proc. Workshop on Scientific Uses of the Space Station in Cosmic Ray and High Energy Gamma Ray Physics, La Jolla, 1985, p. 125 .

9. J.F. Ormes et al., "Report of the Astromag Definition Team", NASA (1988).

10. R.E. Streitmatter et al., Adv. Space Res. 9, 65 (1989)

11. M.H. Salamon et al., Astrophys. J. 349, 78 (1990)

12. J.W. Mitchell et al., Phys. Rev. Lett. 76, 3057 (1996)

13. K. Yoshimura et al., Phys. Rev. Lett. 75, 3792 (1996)

14. J.W. Mitchell et al., Nucl. Phys. Proc. Suppl. 134, 31 (2004)

15. K. Abe et al., Phys. Lett. B670, 103 (2008)

16. K. Abe et al., Phys. Rev. Lett. 108, 051102 (2012)

17. O. Adriani et al., Phys. Rev. Lett. 108, 121101 (2010)

18. A. Yamamoto et al., Adv. Space. Res. in press (2012)

19. S. Orito et al., Phys. Rev. Lett. 84, 1078 (2000)

20. K. Abe et al., Phys. Rev. Lett. 108, 131301 (2012)

21. N. Lund and M. Rothenberg, Astron. Astrophys. 164, 231 (1986)

22. H. Fuke et al., Phys. Rev. Lett. 95, 081101 (2005)

23. J.W. Mitchell et al., Adv. Space Res. 35 (1), 135 (2005) 